\title{
Effects of repeated administration of intradermal skin test by Mantoux method on delayed-type hypersensitivity responses in healthy young and elderly subjects
}

(First published online 6 December 2007)

The elegant and comprehensive review of markers for the evaluation of immunomodulation published in the British Journal of Nutrition ${ }^{(1)}$ highlighted the importance of delayed-type hypersensitivity (DTH) responses as a highly suitable marker of immune function. Because the DTH 'skin test' can predict morbidity and mortality ${ }^{(2,3)}$, it has been suggested as a clinically meaningful test to evaluate the effect of nutritional as well as other types of interventions on the immune response. Previously Multitest CMI, a procedure that involved the simultaneous administration of seven recall antigens was used to evaluate $\mathrm{DTH}^{(4)}$. One advantage of this test was that it did not result in a significant boosting effect upon repeated administration and thus could be used more than once in the same individual. However, this test is no longer commercially available and to our knowledge there is no plan to introduce a similar product to the market. An alternative approach is the standard Mantoux technique in which several antigens (tetanus toxoid, tuberculin, Candida and Trichophyton) ${ }^{(5,6)}$ are administered intradermally, and the diameters of skin induration are read at 24 and $48 \mathrm{~h}$. The Mantoux method may be advantageous because of better sensitivity to detect induration in immunosuppressed patients ${ }^{(7)}$. There is relatively limited information available on the effect of repeated use of this test in healthy subjects. This information is needed since evaluating the effect of nutritional and other interventions to modify the immune response will require repeated administration of the DTH test and a significant boosting effect introduced by the test itself would confound the treatment effect. In order to address this question, we conducted a study to test the magnitude, if any, of the boosting effect due to repeated administration of different antigens by the Mantoux method in young and old healthy subjects.

Ten young (aged 20-40 years) and ten old (aged 66-78 years) free-living men were recruited for this study. Since the magnitude of the DTH skin test is different between men and women ${ }^{(8)}$, only men were recruited in order to reduce gender-associated variation. Exclusion from enrolment in the study included presence of active neoplastic disease; chronic antibiotic use; immunosuppressive drug or glucocorticoid steroid use (prednisone or equivalent $>10 \mathrm{mg} / \mathrm{d}$ ) 1 month prior to or during the study; recent infection ( $\leq 2$ weeks before enrolment); prior positive tuberculin skin test or BCG vaccination; known severe allergy to any kind of food or drug. The Tufts University New England Medical Center Human
Investigation Committee approved the study protocol and informed consent. Each age group of volunteers was randomly assigned to receive either two or three intradermal skin tests. Volunteers were advised to refrain from vaccination, drastic changes in usual dietary intake or activity levels, and intake of micronutrient supplements, and to stop use of nonsteroidal anti-inflammatory drugs $72 \mathrm{~h}$ prior to administration of the test.

Four recall skin test antigens, tuberculin (Tubersol ${ }^{\circledR}$; Aventis Pasteur, Swiftwater, PA, USA), Candida albicans (Can$\operatorname{din}^{\circledR}$, Allermed laboratories, San Diego, CA, USA) and Trichophyton species (Trichophyton mentagrophytes in conjunction with T. rubrum; Hollister-Stier Labs, Spokane, WA, USA), tetanus toxoid (tetanus toxoid USP; Aventis Pasteur, Swiftwater, PA), and a negative control ( $0.9 \%$ normal saline; Bound Tree Medical, Dublin, OH, USA) were injected intradermally in a standard volume of $0.1 \mathrm{ml}$ (except for tetanus toxoid, which was injected in a volume of $0.025 \mathrm{ml}$ ( $0 \cdot 2$ LF units per dose) intradermally at separate sites on the volar surface of the forearm in a clean area free of hair or acneiform rash.

To determine the magnitude of the boosting effect due to repeated administration, the young and old volunteers were divided into two groups. One group ( $n 5$ per age group) received the test antigens and a negative control at 0,1 , and 3 months while the other group ( $n 5$ per age group) received them at 0 and 3 months. The tests were administered and diameters of skin induration were read at 24 and $48 \mathrm{~h}$ by the same investigator (T. A.). The diameter of each induration was measured twice using a metric caliper; the two measurements were averaged and the mean score was recorded. A positive skin test was defined as an induration diameter exceeding $5 \mathrm{~mm}$. The number of positive responses to different antigens, diameter of induration in response to each antigen, and a composite score based on the responses to all antigens were calculated. Data are presented as means and standard errors of the mean, and were analysed using repeated measure two-way ANOVA for the effect of age and repeated administration using Systat 10 software (Systat, Evanston, IL, USA).

The mean age of the young subjects was 29.4 (SE 1.7) years and of the elderly was 71.9 (SE 1.4) years. None of the subjects showed a positive response to the saline injection. At baseline, the young subjects had higher total diameter of induration compared to that of the older subjects (46.6 (SE 4.0) $\mathrm{mm} v$. 
35.6 (SE 6.4) mm; $P=0.064$ ). At 1-month intervals, young subjects also had higher total diameter of induration relative to older subjects (57.9 (SE 5.6) $\mathrm{mm} \mathrm{v.} \mathrm{32.7} \mathrm{(SE} \mathrm{9.8)} \mathrm{mm;}$ $P=0.057$ ). At 3 -month intervals, there was no significant difference in the diameter of indurations between young and older subjects.

No significant effect on the total number of positive antigens was observed after the administration of two or three skin tests in young subjects (data not shown). Young subjects who were administered two skin tests within 3 months of each other (month $0 v$. month 3 ) exhibited a significant increase $(P<0.05)$ in total diameter of induration (total score) and in response to tetanus toxoid (Fig. 1 (A)). Similarly, young subjects who were administered three skin tests at months 0,1 and 3 showed a significant increase $(P<0.05)$ in induration diameter as well as in response to tetanus toxoid and Candida from first to third administration (month $0 v$. month 3 ) (Fig. 1 (C)). In addition, these subjects exhibited a significant increase $(P<0.05)$ in total diameter of induration and response to tetanus toxoid after the second administration (month $0 v$. month 1) (Fig. 1 (C)).

When two tests were administered within 3 months of each other in the elderly volunteers, there was no significant enhancement from baseline in number of positive antigens (data not shown), total diameter of indurations or response to individual antigens (Fig. 1 (B)). Similarly when three tests were administered, with the exception of Trichophyton, there was no significant boosting effect in terms of the number of positive antigens, total score, or response to individual

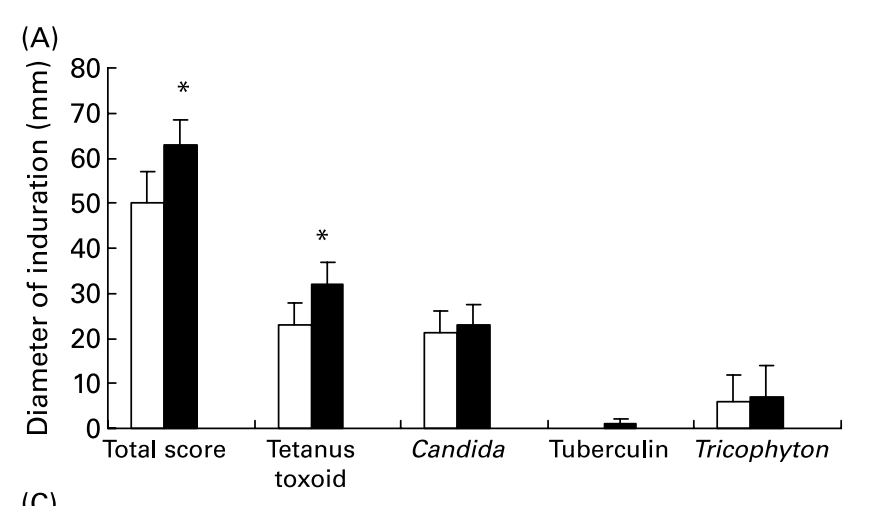

(C)

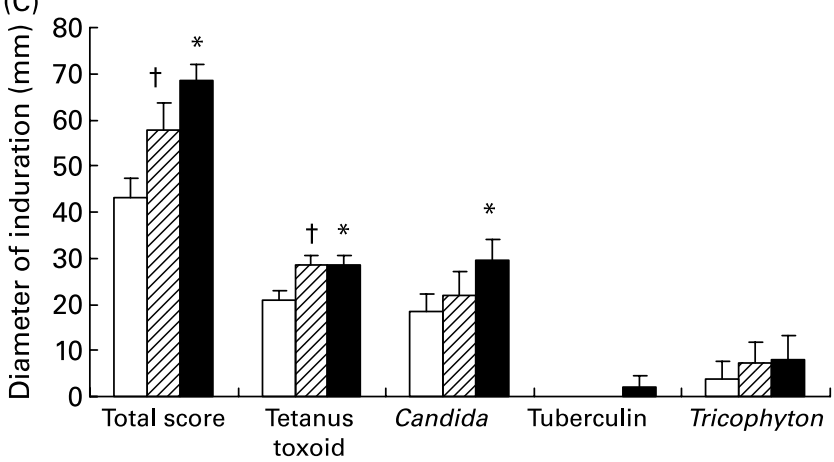

antigens following the second test in the elderly subjects (month $0 v$. month 1) (data not shown for number of positive antigens and Fig. 1 (D)). However, a significant enhancement $(P<0.05)$, from first administration in the number of positive antigens (data not shown), total diameter of induration, and response to the individual antigens tetanus toxoid, Candida, and Trichophyton was observed after the third administration (month $0 v$. month 3) (Fig. 1 (D)).

In summary, we found that the serial administration of DTH skin tests using multiple recall antigens resulted in significant increases in the total diameter of induration in the young at 1 and 3 months whether or not two or three tests were administered consecutively. There was no increase in the average number of positive responses after 1 or 3 months in the young subjects. In the elderly there was not a significant increase in total score at 1 or 3 months if two tests were administered. However, when three tests were performed within 3 months in the elderly subjects, there was a significant increase in total diameter of induration as well as number of positive antigens at 3 months compared to their baseline. Thus, investigators who choose to use the Mantoux test to assess DTH response need to be aware that, in young healthy subjects, there is a significant boosting effect due to the test itself when two or three tests are sequentially administered within a 3 -month period. This boosting effect might confound the results of interventions that are expected to have a modest effect on immune function in young subjects. In contrast, this boosting effect is not observed in the elderly when two tests are administered
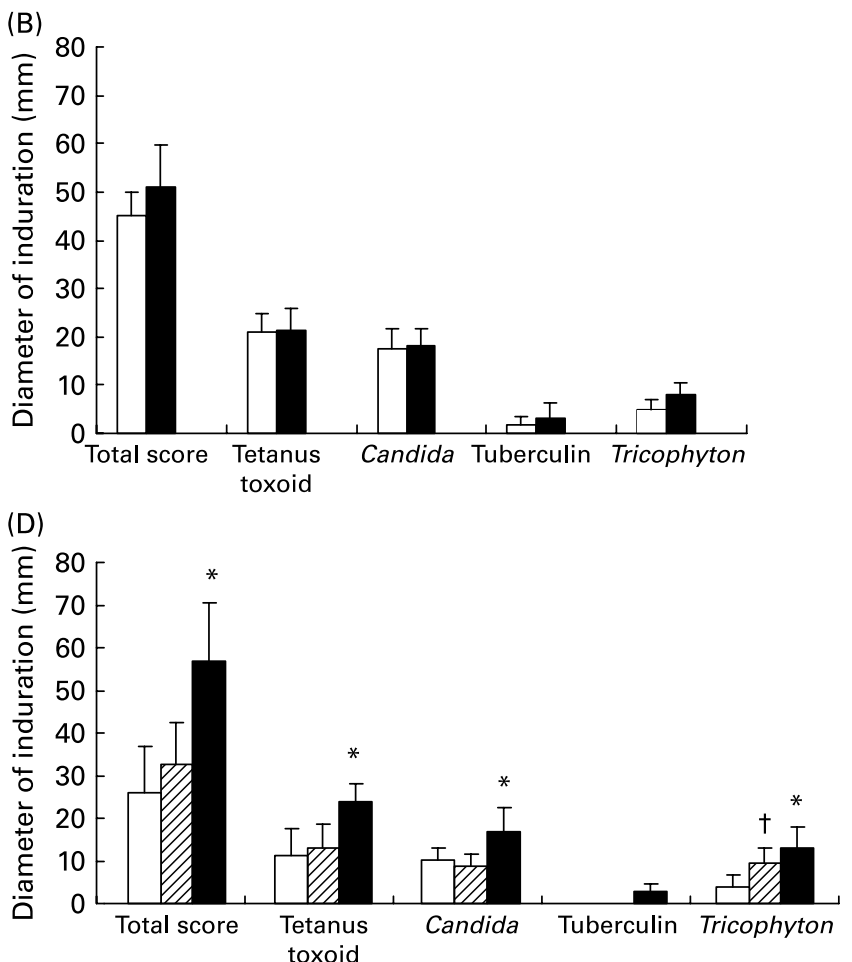

Fig. 1. Delayed-type hypersensitivity responses to four recall antigens following two consecutive administrations (month 0 , $\square$; month 3 , $\mathbf{\square})$ in young (A) and elderly

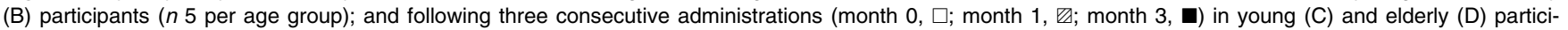
pants ( $n 5$ per age group). Mean values show significant differences between 0 and 3 months: ${ }^{\star} P<0.05$. Mean values show significant differences between 0 and 1 month: $† P<0.05$ 
even within 1 month of each other. However, a significant boosting effect is observed in the elderly when three tests are administered within a 3-month period. Further studies are needed to determine if a period of time longer than 3 months between repeated DTH testing will eliminate the observed boosting effect in young subjects. Previous work has shown that boosting of tuberculin sensitivity is maximal if the interval between the first and second test is between 1 and 5 weeks and is much less frequent if the interval is more than 8 weeks $^{(9)}$. Thus a longer period between the two sequentially administered DTH tests in young subjects might overcome this problem.

\section{Acknowledgements}

Supported by USDA contract no. 58-1950-7-707 and an Ellison Medical Foundation-International Nutrition Foundation fellowship. The authors thank the staff of JMUSDA-HNRCA Metabolic Research Unit for their help with subject recruitment and conduct of the study.

Tanvir Ahmed ${ }^{1,2}$ Davidson H. Hamer ${ }^{1,3,4}$ Simin Nikbin Meydani ${ }^{1,5}$

${ }^{1}$ Nutritional Immunology Laboratory, Jean Mayer USDA Human Nutrition Research Center on Aging at Tufts University, Boston, MA 02111, USA

${ }^{2}$ International Centre for Diarrhoeal Disease Research, Dhaka, Bangladesh

${ }^{3}$ Center for International Health and Development, Boston University School of Public Health, Boston, MA, USA ${ }^{4}$ Section of Infectious Diseases, Department of Medicine, Boston University School of Medicine, Boston, MA, USA
${ }^{5}$ Friedman School of Nutrition, Science and Policy, Department of Pathology, Sackler Graduate School of Biochemical Sciences, Tufts University, Boston, MA 02111, USA simin.meydani@tufts.edu

\section{References}

1. Albers R, Antoine J, Bourdet-Sicard R, et al. (2005) Markers to measure immunomodulation in human nutrition intervention studies. Br J Nutr 94, 452-481.

2. Christou NV, Tellado-Rodriguez J, Chartrand L, Giannas B, Kapadia B, Meakins J, Rode H \& Gordon J (1989) Estimating mortality risk in preoperative patients using immunologic, nutritional, and acute-phase response. Ann Surg 210, 69-77.

3. Wayne SJ, Rhyne RL, Garry PJ \& Goodwin JS (1990) Cellmediated immunity as a predictor of morbidity and mortality in subjects over 60. J Gerontol 45, M45-M48.

4. Lesourd BM, Wang A \& Moulias R (1985) Serial delayed cutaneous hypersensitivity skin testing with multiple recall antigens in healthy volunteers: booster effect study. Ann Allergy 55, 729-735.

5. Grabenstein GD (2002) Immunofacts: Vaccines and Immunologic Drugs. St. Louis: Wolters Kluwer Company.

6. England R, Nugent J, Grathwohl K, Hagan L \& Quinn J (2003) High-dose inhaled fluticasone and delayed hypersensitivity skin testing. Chest 123, 1014-1017.

7. Martinez-Marcos FJ, Lopez-Cortes LF, Pachon J, Alarcon A, Cordero E \& Viciana P (1998) Comparison of two methods for the assessment of delayed-type hypersensitivity skin responses in patients with human immunodeficiency virus infection. Clin Infect Dis 26, 1330-1334.

8. Vine M, Stein L \& Weigle K (2000) Gender differences in response to the multitest CMI skin test in the general population. Ann Allergy Asthma Immunol 84, 445-450.

9. Cauthen GM, Snider DE Jr \& Onorato IM (1994) Boosting of tuberculin sensitivity among Southeast Asian refugees. Am J Respir Crit Care Med 149, 1597-1600. 